\title{
La datation des archives glaciaires
}

\author{
Frédéric Parrenin \\ Université Grenoble Alpes, CNRS, IRD, IGE, Grenoble, France
}

Amaëlle Landais

Laboratoire des Sciences du Climat et de l'Environnement, CEA/CNRS/UVSQ, Saclay

\section{$\underline{\text { Résumé }}$}

Les glaces en Antarctique et au Groenland sont une archive de premier plan pour reconstruire le climat du Quaternaire. Une datation précise est nécessaire pour exploiter cette archive. Les méthodes de datation peuvent être tirées de l'analyse de la glace, des bulles d'air piégées, de la comparaison à d'autres archives ou aux variations des paramètres orbitaux de la Terre, ou de la modélisation du processus de sédimentation glaciaire. Ces méthodes étant complémentaires, un modèle probabiliste a été développé pour les combiner de manière optimale.

\section{The dating of glacial archives}

\section{Summary}

Ice samplings from Antarctica or Greenland form an exceptional archive to reconstruct the Quaternary climate. An accurate chronology is necessary to exploit this archive. Dating methods can be derived from the analysis of ice, from enclosed air bubbles, from the comparison to other archives or to variations of astronomical parameters of the Earth, or from the modelling of the glacial sedimentation process. These methods being complementary, a probabilistic model has been developed to combine them in an optimal manner.

\section{Introduction}

La richesse du témoignage qu'apportent les grands forages glaciaires de l'Antarctique et du Groenland (Figure 1) sur les variations passées de notre climat et de notre environnement est reconnue bien au-delà de la communauté des glaciologues. De façon unique, les glaces permettent de reconstituer, à partir d'une même archive, les variations locales du climat et celles de la composition de l'atmosphère, qui elles sont pour partie globales. L'exploitation des informations qu'offrent les archives glaciaires nécessite la datation aussi précise que possible de ces différents enregistrements. Là aussi, la glace a ses spécificités.

La première tient au fait que la glace résulte du tassement des couches de neige sous leur propre poids. En surface, la neige est très peu dense (entre 0,3 et $0,4 \mathrm{~g} / \mathrm{cm}^{3}$ ) : l'air circule librement dans les premiers mètres de ce milieu poreux, le névé, puis plus difficilement en profondeur, car la densité augmente et la porosité diminue. Quand cette densité est supérieure à environ $0,83 \mathrm{~g} / \mathrm{cm}^{3}$ (à partir de 100 mètres de profondeur au centre de l'Antarctique), l'air est piégé dans la glace à l'intérieur de bulles et isolé de l'atmosphère. En profondeur, sous l'effet de la pression, ces bulles se compriment puis se transforment en clathrates, c'est-à-dire que les molécules de gaz sont intégrées à la structure cristalline de la glace. Finalement, l'air est plus jeune que la glace qui l'emprisonne. Pour dater les archives glaciaires, dont certains des signaux sont inscrits dans la glace, d'autres dans les bulles d'air, nous avons donc besoin de deux chronologies distinctes.

Par ailleurs, les glaces ne se prêtent pas à l'utilisation de méthodes radioactives comme le carbone-14 ou la méthode Uranium-Thorium. La datation au carbone-14 ne peut être utilisée 
qu'exceptionnellement, par exemple quand on trouve des débris végétaux ou que l'on extrait des quantités suffisantes de dioxyde de carbone. Même si les quantités de glace nécessaires à la datation au carbone-14 ont diminué depuis l'avènement de la spectrométrie de masse par accélérateur, les dates obtenues ne sont que des valeurs moyennes sur quelques mètres de glace. De plus, cette méthode est inapplicable au-delà de quelques dizaines de milliers d'années en raison de la décroissance radioactive du carbone-14 dont la demi-vie est de 5730 ans.
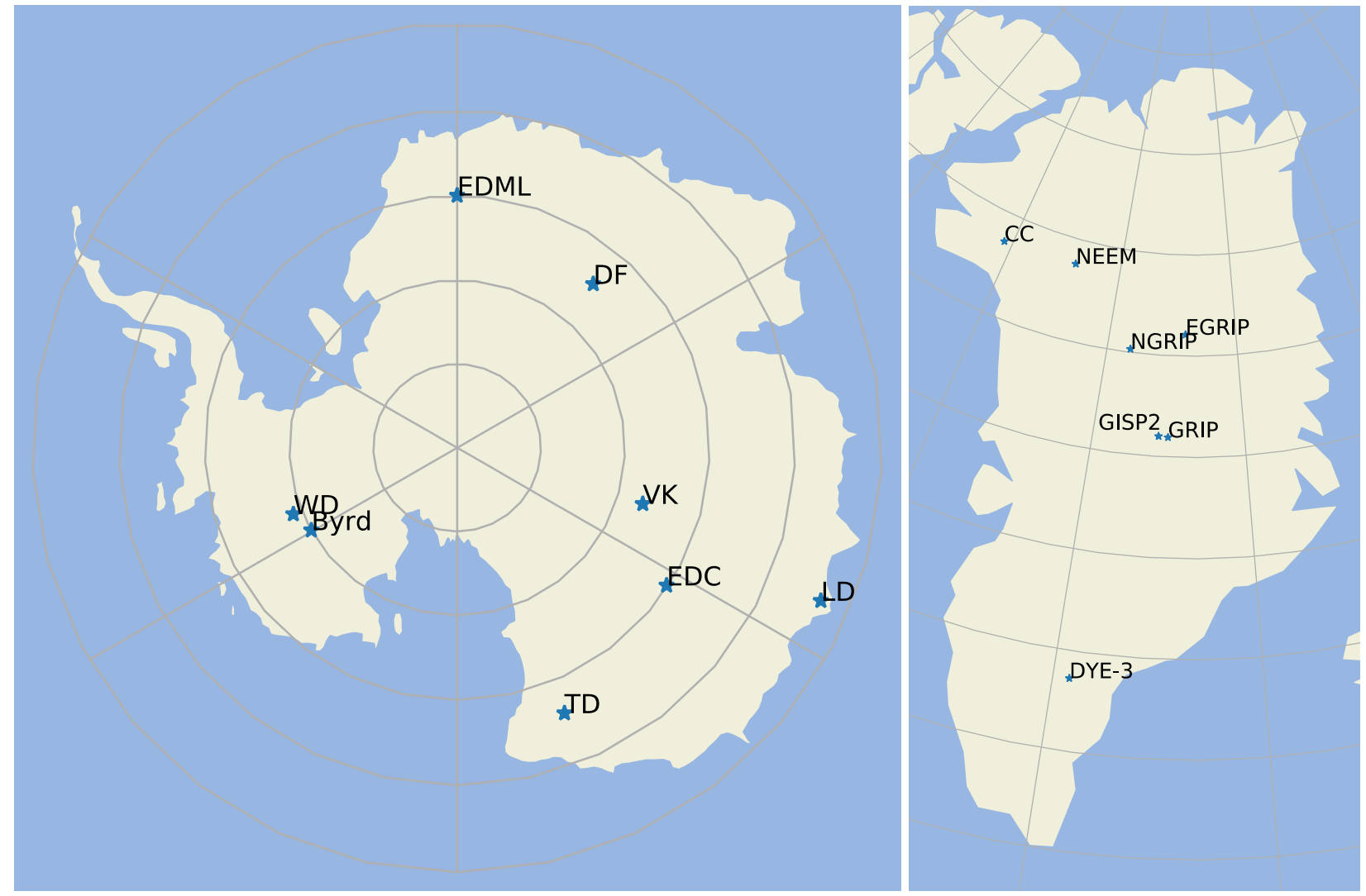

Figure 1 : Cartes des principaux forages. Gauche, Antarctique : EPICA Dome C (EDC), Vostok (VK), Dome Fuji (DF), WAIS Divide (WD), EPICA Dronning Maud Land (EDML), Talos Dome (TD), Law Dome (LD), Byrd. Droite, Groenland : GRIP, GISP2, DYE-3, Camp Century (CC), NorthGRIP (NGRIP), NEEM, EastGRIP (EGRIP)

Les datations mises au point par les glaciologues sont alors fondées sur des méthodes complémentaires à partir des analyses sur la glace (section 2), des analyses sur l'air piégé (section 3), de la comparaison avec d'autres enregistrements datés (section 4) ou avec les variations des paramètres orbitaux (section 5), mais aussi de la modélisation du processus de sédimentation (modélisation de l'accumulation de la neige, du piégeage de l'air et de l'écoulement de la glace, section 6). L'objectif de cet article sera de décrire ces différentes méthodes de datation. Ensuite, nous décrirons en section 7 une technique probabiliste, communément appelée " méthode inverse ", qui consiste à regrouper ces différentes sources d'information chronologique afin d'obtenir une datation optimale et d'en évaluer l'intervalle de confiance. 


\section{Datation par les mesures dans la glace}

\subsection{Le comptage des couches annuelles}

Sur les calottes polaires et sur les glaciers, nombre des propriétés de la neige diffèrent selon qu'elle s'accumule l'été ou l'hiver. Par exemple, l'été, les poussières sont plus abondantes dans la neige car, pendant cette saison, les vents sont favorables au transport des poussières vers les pôles. On peut donc reconnaître des couches annuelles soit visuellement, soit par analyse chimique, soit par analyse isotopique. Le comptage des couches annuelles est ainsi une méthode simple de datation, pourvu que l'accumulation de neige soit suffisante afin que la stratigraphie ne soit pas détruite par l'action du vent qui mélange les couches près de la surface. Pour cette raison, le comptage des couches est impossible dans les régions centrales du plateau Antarctique où sont situés les forages profonds de Vostok, du Dôme C et du Dôme Fuji, mais il l'est sur l'ensemble du Groenland et, pour l'Antarctique, dans les régions plus côtières.

Le Greenland Ice Core Chronology 2005 (GICC05), grand projet de comptage systématique et réalisé par l'équipe danoise du Niels Bohr Institute à Copenhague, est basé sur l'analyse des carottes de DYE-3, GRIP et NorthGRIP, et s'étend actuellement sur les 60000 dernières années (Svensson et al., 2008). Un travail similaire a été effectué sur des carottes à taux d'accumulation suffisamment élevé en Antarctique (WAIS Divide et Pôle Sud) et au Groenland (EGRIP).

Les glaciologues ont recours à divers enregistrements saisonniers pour identifier des couches annuelles (Figure 2). Aucun des indicateurs n'est parfait, mais, combinés, ils autorisent une datation annuelle tant que l'épaisseur des couches, qui s'amincissent en s'enfonçant dans la calotte, reste suffisante. Pour GICC05, le comptage a été effectué par différentes personnes et sur différentes carottes, et les résultats obtenus indépendamment sont comparés de façon à minimiser les erreurs d'appréciation. D'autre part, chaque couche incertaine a été répertoriée de façon à obtenir un intervalle de confiance sur la chronologie finale. Les erreurs répertoriées sont faibles : inférieures à $2 \%$ jusqu'à la dernière déglaciation (18 000 à 11000 ans dans le passé), et de l'ordre de 5\% au-delà.

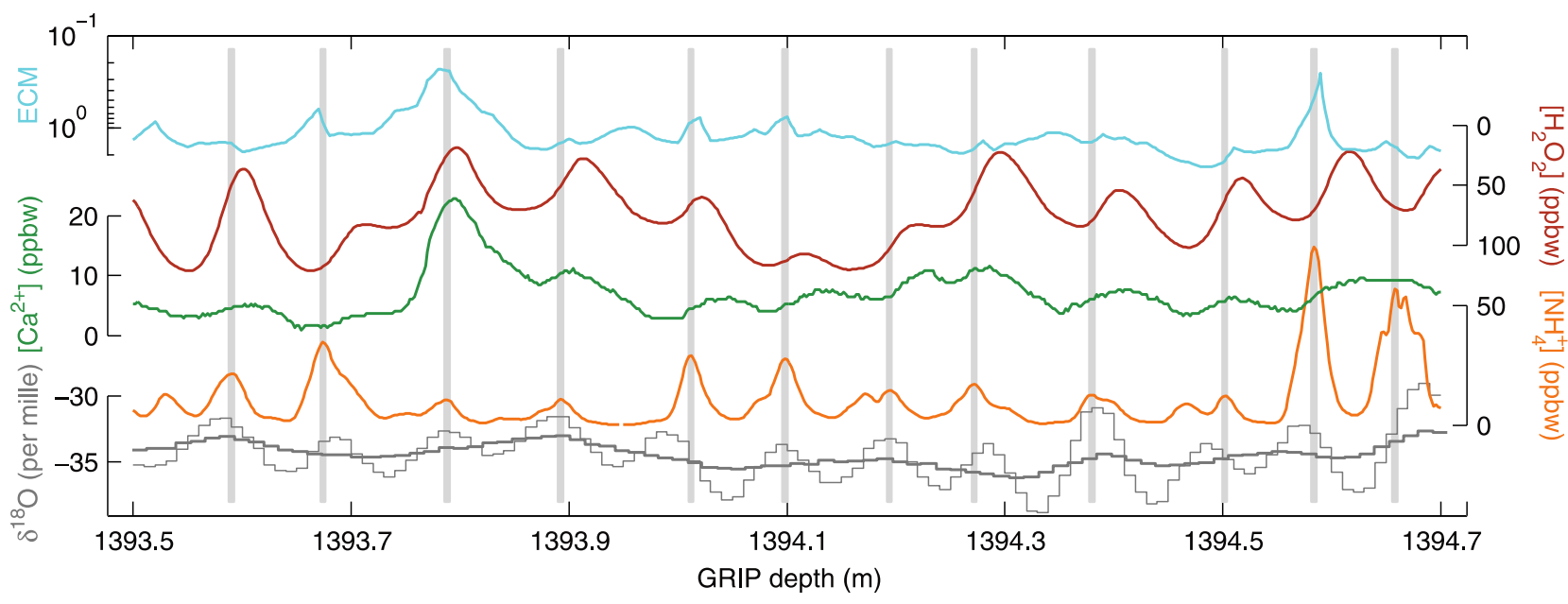

Figure 2 : Exemple d'une section de 1,2 m de la carotte GRIP d'un âge d'environ 8.8 ka avec les couches annuelles marquées par des barres verticales grises. De haut en bas, les enregistrements utilisés pour repérer les couches annuelles sont : ECM, eau oxygénée $\left(\mathrm{H}_{2} \mathrm{O}_{2}\right), \mathrm{Ca}^{2+}, \mathrm{NH}_{4}{ }^{+}$et $\delta^{18} \mathrm{O}$. Pour ce dernier indicateur, la ligne épaisse représente les données brutes et la ligne fine les données après correction de l'effet de diffusion. Extrait de Rasmussen et al. (2006). 


\subsection{Les horizons volcaniques}

Des horizons volcaniques peuvent être repérés dans les forages, aussi bien en Antarctique qu'au Groenland. Quelques événements sont suffisamment intenses ou se produisent suffisamment près du forage pour déposer des poussières (cendres) volcaniques visibles à l'œil nu. La plupart ne déposent que des aérosols de petite taille. On peut alors les repérer grâce aux analyses chimiques effectuées sur la glace : le sulfate en particulier présente de nombreux pics correspondant à des apports volcaniques qui sont facilement repérables car ils dépassent largement les niveaux usuels. Ces horizons volcaniques modifient également l'acidité de la glace, ce qui permet de les repérer en mesurant sa conductivité.

Pour les derniers millénaires, bon nombre de ces événements volcaniques sont bien datés par différentes méthodes (Sigl et al., 2014) : 1) soit par le comptage des couches pour les sites à suffisamment forte accumulation, 2) soit par des écrits historiques, 3) soit par datation sur du matériel volcanique proche du volcan en question, notamment par la méthode carbone-14 appliquée à des débris biologiques (par exemple, les arbres pris dans les laves). À partir d'une datation glaciologique approximative, ces événements peuvent en général être identifiés dans les carottes grâce à leur intensité. Ils permettent également de relier stratigraphiquement deux forages (voir Figure 3).

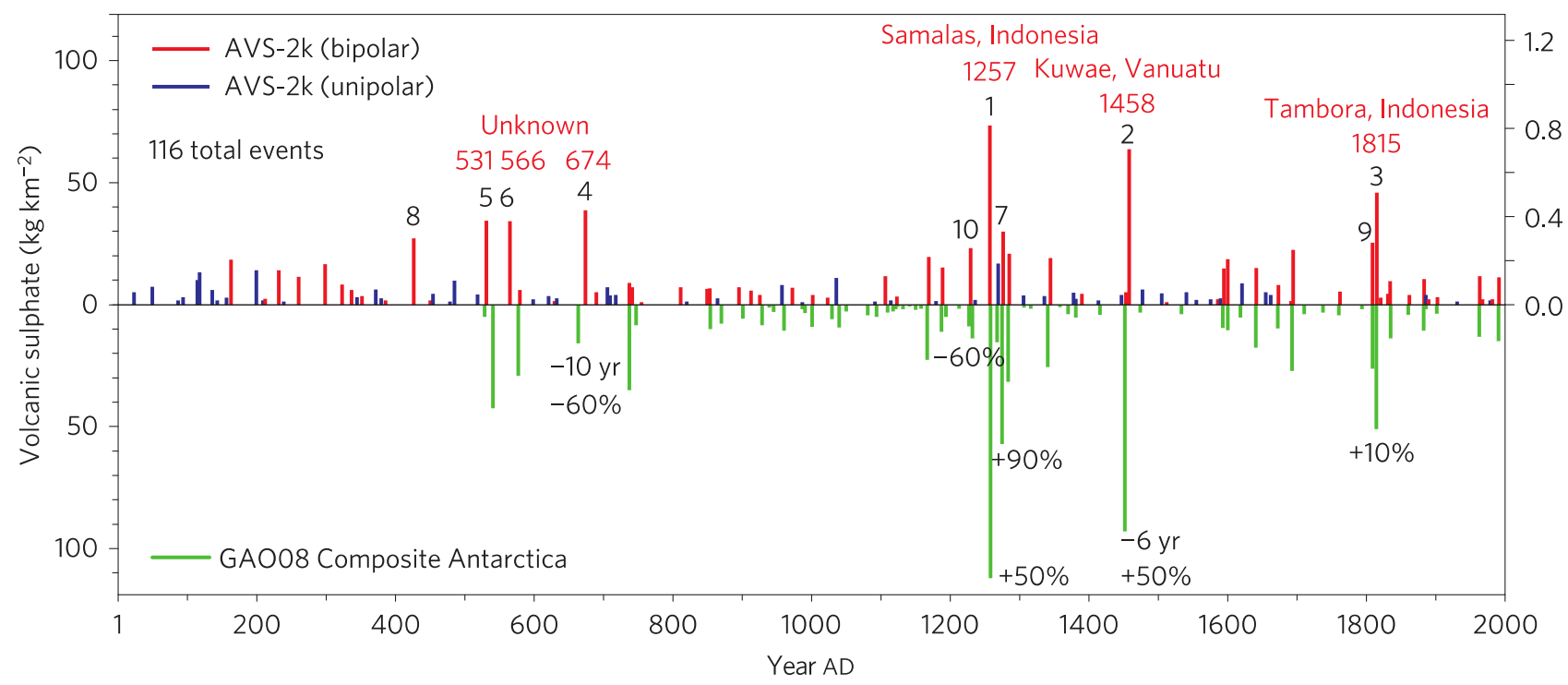

Figure 3 : Intensité des éruptions volcaniques des derniers 2000 ans. Les éruptions impactant l'hémisphère nord sont données en bleu, celles de l'hémisphère sud en vert, et les éruptions globales sont indiquées en rouge. Extrait de Sigl et al. (2014).

Au-delà de l'Holocène (les derniers 11000 ans), seules quelques couches de cendres peuvent être datées précisément. L'analyse chimique de ces cendres permet sans ambiguïté l'identification de l'éruption volcanique concernée. Cette signature peut parfois être mise en relation avec celle de matériel volcanique présent à proximité d'un volcan. Ce matériel volcanique, en plus grande quantité, peut lui être daté par des méthodes radiochronologiques classiques.

\subsection{Les variations du champ magnétique et de l'activité solaire}

Le ${ }^{10} \mathrm{Be}$ et le ${ }^{14} \mathrm{C}$ sont tous deux produits dans la haute atmosphère par le flux de particules cosmiques. Ce flux est modulé d'une part par le champ magnétique du vent solaire qui dévie les particules chargées et d'autre part par le champ magnétique terrestre. À la différence du ${ }^{10} \mathrm{Be}$ dont le flux de dépôt à la surface de la Terre est pratiquement directement lié à la production dans la haute atmosphère, la composition en ${ }^{14} \mathrm{C}$ de l'atmosphère est également modulée par les échanges entre les 
différents réservoirs de carbone sur Terre. Mais les variations majeures de ces deux indicateurs $\left({ }^{14} \mathrm{C}\right.$ et ${ }^{10} \mathrm{Be}$ ) sont concomitantes.

Le ${ }^{10} \mathrm{Be}$ peut être mesuré avec précision dans les carottes de glace, aussi bien en Antarctique (Raisbeck et al., 2007 et références incluses) qu'au Groenland (Beer et al., 2006 et références incluses). On importe ainsi la datation par comptage des couches annuelles du Groenland sur les carottes Antarctique pour l'Holocène ou pour l'anomalie du champ magnétique terrestre de Laschamp survenue il y a environ 41000 ans (Raisbeck et al., 2007).

Le ${ }^{14} \mathrm{C}$, quant à lui, est mesuré dans les anneaux des arbres, qui sont datés très précisément pour les derniers 12550 ans grâce à la dendrochonologie (Reimer et al., 2013). On transpose ainsi cette datation sur les carottes de glace lorsque les variations de l'activité solaire sont suffisamment importantes pour synchroniser ${ }^{10} \mathrm{Be}$ et ${ }^{14} \mathrm{C}$. Cette méthode a été utilisée pour dater la partie Holocène des carottes de glace Antarctique pour lesquelles le comptage des couches n'est pas possible.

Enfin, les anomalies importantes du champ magnétique terrestre peuvent être repérées dans d'autres archives paléoenvironnementales comme les laves volcaniques qui peuvent être datées par les méthodes basées sur l'argon/argon ou sur le potassium/argon. L'anomalie de Laschamp est ainsi datée avec une relativement bonne précision (Guillou et al., 2004). La transition de BruhnesMatuyama plus ancienne (il y a environ 790000 ans) a fait l'objet d'études récentes pour fournir la datation la plus précise possible (Sagnotti et al., 2016).

\section{Datation par les mesures dans les bulles d'air}

\subsection{Une complication : la différence d'âge entre la glace et l'air}

Le névé est la zone supérieure des calottes qui est poreuse. Elle marque la transition entre la neige qui tombe en surface, et la glace en profondeur. Selon les sites et en particulier les conditions de température et d'accumulation en surface, son épaisseur varie grossièrement de $50 \mathrm{~m}$ (Groenland) à $120 \mathrm{~m}$ (Antarctique central). Sa densité varie entre la densité de surface (typiquement $0.4 \mathrm{~g} / \mathrm{cm}^{3}$ ) et la densité à la profondeur de fermeture, c'est-à-dire la profondeur à laquelle les pores se ferment (typiquement $0.83 \mathrm{~g} / \mathrm{cm}^{3}$ ). À cette profondeur, où l'on considère le stade glace atteint, l'air est piégé dans des bulles isolées et ne circule plus.

L'étude du transport des gaz dans le névé a conduit à l'élaboration d'un modèle simple (Sowers et al., 1992) à partir duquel nous pouvons distinguer quatre zones dans le névé (Figure 4) :

- La zone convective, située juste en dessous de la surface. La convection dans cette zone résulte d'une part du gradient thermique, d'autre part de l'action des vents de surface.

L'épaisseur de cette zone est en général très faible (moins de $5 \mathrm{~m}$ ) mais pourrait atteindre 20 $\mathrm{m}$ pour des sites présentant un fort vent, des reliefs en surface (dunes) et des fractures larges dans la structure du névé permettant à l'air de se mélanger.

- La zone diffusive correspond à une zone où la colonne d'air est statique (sans convection). Le mouvement se fait alors à l'échelle moléculaire (diffusion) et on observe dans cette zone des fractionnements élémentaires et isotopiques. Par exemple, les molécules les plus lourdes se déplacent préférentiellement vers les zones froides et basses, tandis que les molécules légères se situent davantage vers les zones chaudes et élevées.

- La zone non diffusive, à l'intérieur de laquelle les pores sont presque fermés et où la diffusion moléculaire devient alors négligeable. La composition des bulles n'est alors plus modifiée, bien que la pression totale puisse encore évoluer.

- La zone de fermeture des bulles, ou zone de fermeture, se situe à la base du névé. Les bulles de gaz se ferment et l'air est alors piégé à l'intérieur. Comme mentionné plus haut, la 
fermeture correspond à une densité voisine de $0.83 \mathrm{~g} / \mathrm{cm}^{3}$.

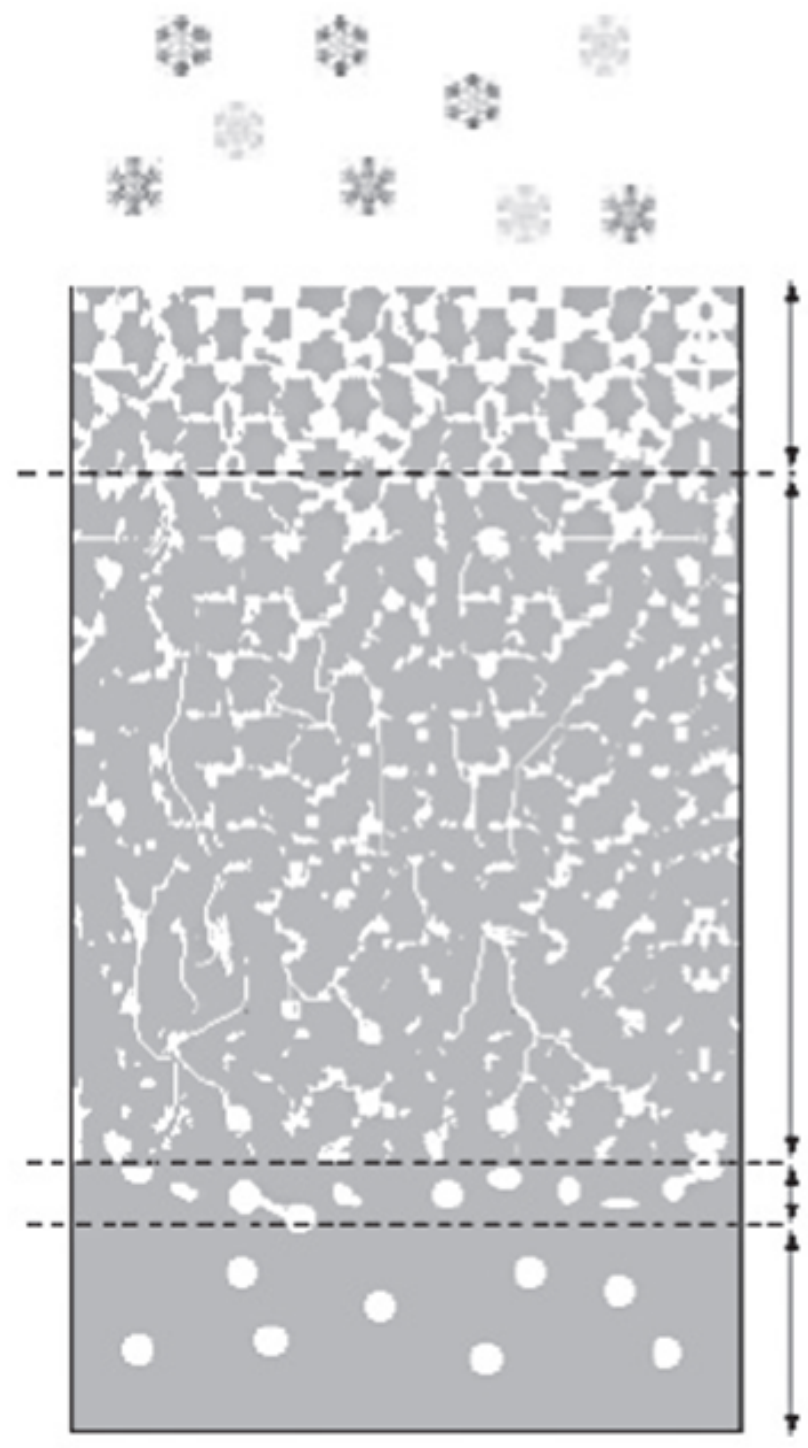

\section{accumulation}

zone convective

\section{zone diffusive}

\section{zone non-diffusive zone de fermeture}

\section{glace}

Figure 4 : Schéma illustrant le métamorphisme progressive de la neige en glace dans un névé. Adapté de Sowers (1992).

Le sommet de la zone non diffusive correspond à une profondeur critique au-dessus de laquelle l'air contenu dans l'espace poreux du névé est encore en contact avec l'atmosphère de surface. Alors que la neige, à cette profondeur, s'est déposée depuis plusieurs centaines ou même milliers d'années, l'air, lui, en est encore quasiment à l'âge « zéro », c'est-à-dire l'âge de la neige à la surface. Par conséquent, à n'importe quelle profondeur d'une carotte de glace, l'air contenu dans la bulle d'air piégée est toujours plus jeune que la glace qui l'entoure. La différence d'âge entre la glace et les bulles d'air est appelée et notée $\Delta$ âge. Dit autrement, on trouve de l'air ayant le même âge que la glace à une profondeur plus basse, cette différence de profondeur étant appelée $\Delta$ profondeur (ou $\Delta$ prof en abrégé). 


\section{Les événements de Dansgaard-Oeschger}

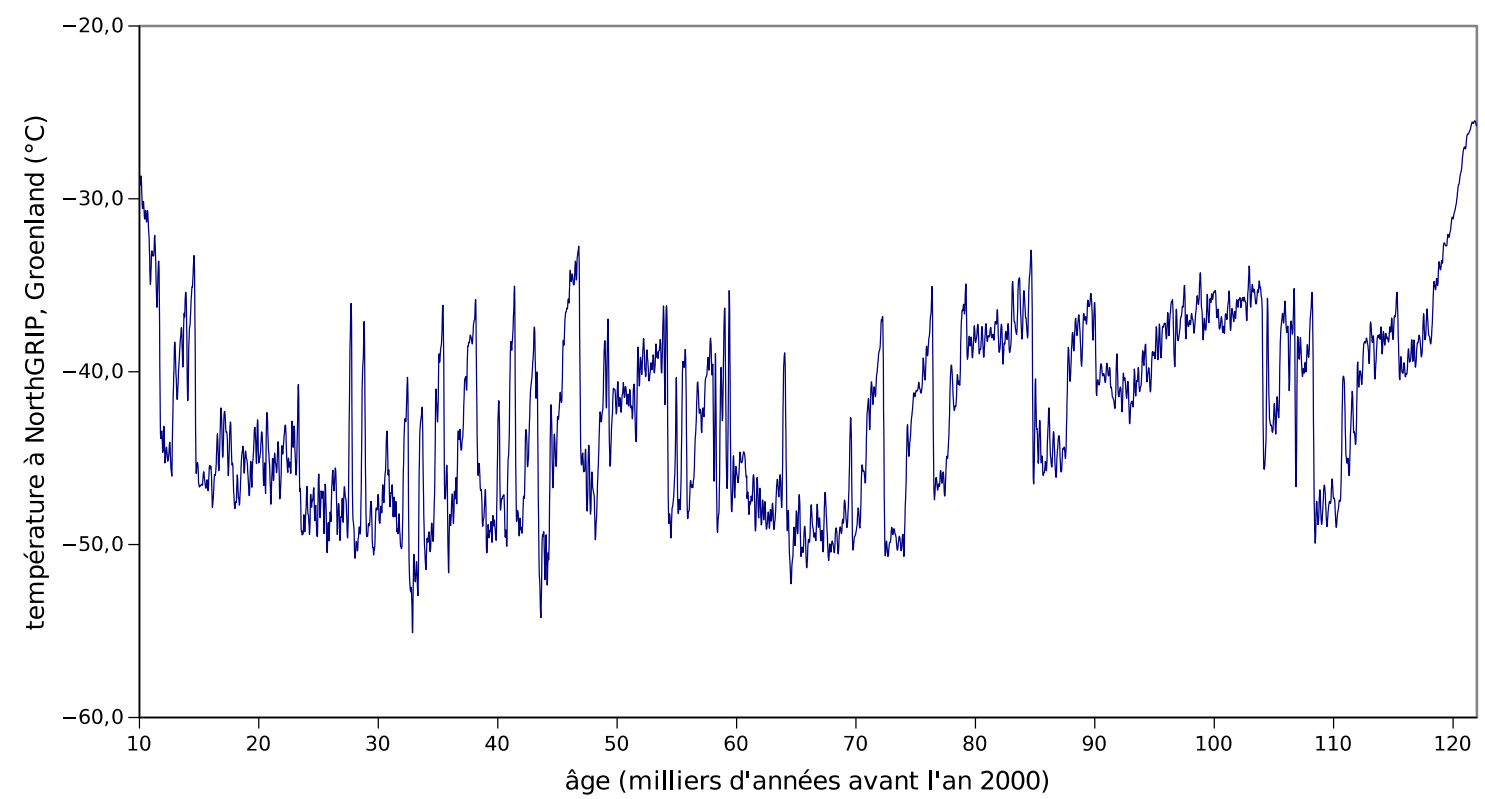

Les événements de Dansgaard-Oeschger (D-O) sont des variations rapides du climat survenues lors des dernières périodes glaciaires. Au Groenland, les D-O prennent la forme d'un réchauffement rapide (de 5 à $16^{\circ} \mathrm{C}$ ) survenu en quelques décennies, suivi d'un refroidissement plus graduel. Les phases froides des D-O sont appelés les stades, et les phases chaudes les interstades. La durée typique d'un cycle D-O est très variable, de 200 ans à plus de 3000 ans.

Bien qu'initialement découverts dans les glaces du Groenland, on retrouve des événements comparables en forme et en âge aux D-O dans une grande partie de l'hémisphère nord mais avec une amplitude pour le changement de température plus faible aux plus basses latitudes. Ces changements sont aussi associés à des variations du cycle hydrologique comme l'indique les enregistrements sur les spéléothèmes des grottes en Chine. A partir des glaces d'Antarctique, on a pu identifier qu'à chaque événement de D-O, un événement est associé (Maximum Isotopique d'Antarctique). En Antarctique, les changements de température sont plus graduels et asynchrones à ceux du Groenland.

Les causes et mécanismes des D-O ne sont à l'heure actuelle pas totalement établis. On sait que la circulation océanique, notamment dans l'Atlantique nord, est susceptible de changer brusquement de mode de fonctionnement. Des purges des calottes polaires de l'hémisphère nord, enregistrées dans les sédiments marins, ont pu jouer un rôle sur cette circulation océanique.

\subsection{La synchronisation Groenland-Antarctique par le méthane}

La connaissance du décalage glace-air permet de transférer des informations chronologiques de l'air vers la glace. Par exemple, des variations caractéristiques du méthane des bulles d'air permettent de synchroniser différentes carottes de glace, même issues de pôles différents. Ainsi, les événements de Dansgaard-Oeschger (D-O) ont été repérés pour la première fois dans les carottes de glace du Groenland et correspondent à des variations abruptes de température durant la dernière période glaciaire (Johnsen et al., 1992). Des variations synchrones (à quelques dizaines d'années près) sont également observées dans la teneur atmosphérique en méthane (EPICA community members, 2006) que l'on mesure dans les bulles d'air aussi bien dans la glace de l'Antarctique que dans celle du Groenland. Le méthane mesuré dans l'air est donc un puissant outil de 
synchronisation.

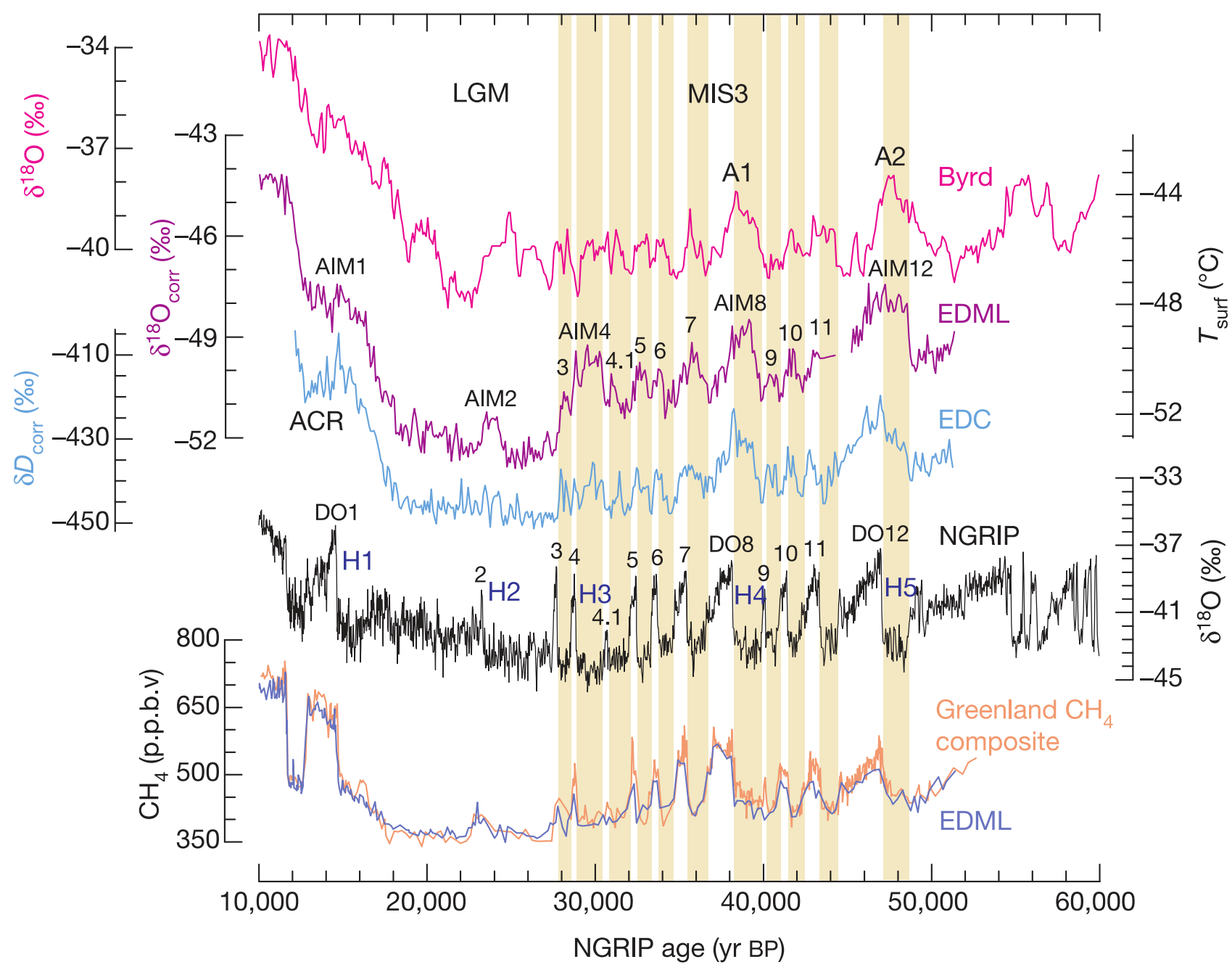

Figure 5 : Synchronisation méthane et explication de la bascule bipolaire. Les 3 courbes en haut représentent les variations isotopiques de la glace enregistrées dans 3 forages antarctique : Byrd, EDML et EDC. La courbe en noir au milieu représente les variations isotopiques de la glace au Groenland à partir du forage NGRIP. Les deux courbes du bas représentent le méthane enregistré au Groenland et à EDML qui a permis la synchronisation. La bascule bipolaire décrit le fait que sur la variabilité millénaire à centennale de la dernière période glaciaire, l'Antarctique se réchauffe (resp. se refroidit) pendant les phases froides (resp. chaudes) du Groenland. Extrait de EPICA community members (2006).

\subsection{Les méthodes ${ }^{40} \mathrm{Ar}$ et ${ }^{81} \mathrm{Kr}$}

La décroissance radioactive $\mathrm{du}^{40} \mathrm{~K}$ depuis le manteau terrestre conduit à une augmentation de la concentration de ${ }^{40} \mathrm{Ar}$ dans l'atmosphère. En mesurant le rapport isotopique ${ }^{40} \mathrm{Ar} /{ }^{36} \mathrm{Ar}$ (exprimé sous la notation $\delta^{40} \mathrm{Ar}$ ), il est ainsi possible d'avoir une estimation de l'âge de l'air piégé dans les bulles. À partir de la mesure du $\delta^{40} \mathrm{Ar}$ dans des bulles d'air datées par ailleurs dans la carotte d'EDC, on a pu estimer un taux d'augmentation d'environ 0.066 \% par million d'années (Bender et al., 2008), ce qui reste très faible comparé à la précision des mesures de l'ordre de $0.020 \%$ du $\delta^{40} \mathrm{Ar}$ piégé dans les bulles d'air qui est aussi affecté par les fractionnements thermiques et gravitationnels décrits plus haut. L'erreur associée à chaque détermination d'âge reste donc importante et de l'ordre de 100000 à 200000 ans au minimum. La méthode devient aussi problématique pour les glaces situées à proximité du lit rocheux (les plus anciennes), où $~^{140} \mathrm{Ar}$ peut être rajeuni (augmenté) par les 
dégagements du socle rocheux à proximité.

La demi-vie du ${ }^{81} \mathrm{Kr}$ est de 229000 ans (c'est-à-dire qu'il faut 229000 ans pour que sa concentration décroisse de moitié). La mesure de sa concentration atmosphérique en fait donc un outil particulièrement intéressant pour la datation des carottes de glace ancienne, jusqu'à 2 millions d'années que la communauté des glaciologues prévoit de forer. La concentration de ${ }^{81} \mathrm{Kr}$ dans l'atmosphère est cependant très difficile à mesurer car le ${ }^{81} \mathrm{Kr}$ est très peu abondant dans l'atmosphère. La technique analytique utilisée pour ces mesures est une technique de comptage d'atomes individuels utilisant un piège magnéto-optique (Atom Trap Trace Analysis). Les premières mesures nécessitaient plusieurs dizaines de kilos de glace. Elles ont donc été effectuées sur la carotte horizontale de Taylor Dome où de la glace vieille de plus de 100000 ans affleure en surface (Buizert et al., 2014). Depuis quelques années, la quantité de glace nécessaire a été considérablement réduite. Une quantité de $6 \mathrm{~kg}$ permet dorénavant d'atteindre une incertitude de $10 \%$ sur la détermination de l'âge absolu.

\section{Datation par synchronisation sur d'autres archives : l'exemple des spéléothèmes}

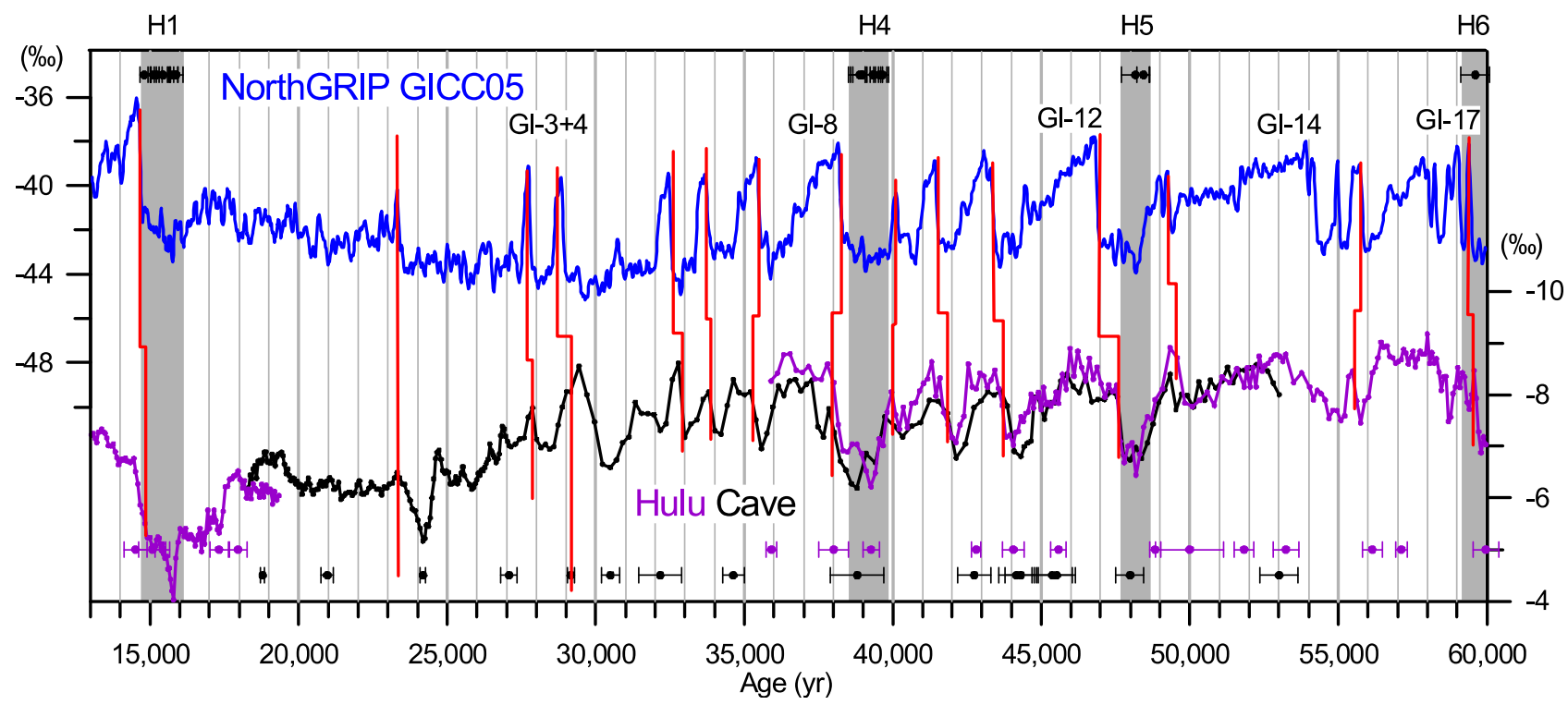

Figure 6 : Les événements de Dansgaard Oeschger (GI, Greenland Interstadial) repérés dans la carotte de NorthGRIP (courbe bleue, datation GICC05) et dans les enregistrements de la grotte Hulu (courbes noire et violette, Wang et al., 2001). Les points datés des enregistrements d'Hulu Cave par la méthode Uranium/Thorium sont marqués en bas de la figure (avec leur barre d'erreur). Les zones grisées représentent les événements de Heinrich tels que repérés dans les spéléothèmes du Brésil (Wang et al., 2004). Leurs points datés par la méthode Uranium/Thorium sont également indiqués en haut de la figure. Extrait de Svensson et al. (2008).

Les variations climatiques des événements de Dansgaard-Oeschger, même si leur amplitude était probablement maximale au niveau de l'Atlantique Nord, sont en fait visibles à de nombreux endroits de la planète dans d'autres archives climatiques. On les retrouve ainsi dans la composition isotopique des spéléothèmes (concrétions calcaires que l'on retrouve dans les grottes) en Europe et en Asie mais aussi en Amérique du Sud et dans l'océan indien. Ces spéléothèmes peuvent être datées avec une précision de l'ordre de quelques centaines d'années à 1 ou 2 milliers d'années sur les derniers cycles climatiques grâce à la méthode Uranium/Thorium. Ils fournissent donc un âge précis pour les transitions marquant les événements D-O et peuvent être utilisés comme référence 
chronologique si l'hypothèse est faite d'un synchronisme entre les variations enregistrées dans le $\delta^{18} \mathrm{O}$ de la calcite des spéléothèmes des basses latitudes (principalement Asie du Sud Est pour les mieux datés) et les variations de méthane, principalement émis pas les zones humides des basses latitudes pendant les périodes glaciaires. La figure 6 montre un exemple de synchronisation potentielle entre la carotte de NorthGRIP et les spéléothèmes de la grotte Hulu en Chine. Cette hypothèse et la synchronisation entre $\delta^{18} \mathrm{O}$ de la calcite de spéléothèmes asiatiques et variations de $\mathrm{CH}_{4}$ enregistrées dans les bulles d'air ont été largement utilisées pour obtenir la datation de la carotte de WAIS au-delà du comptage de couches pendant la dernière période glaciaire (Sigl et al., 2016).

Récemment, il a été suggéré à l'aide de modélisation du climat incluant les isotopes que les variations du $\delta^{18} \mathrm{O}$ des bulles d'air $\left(\delta^{18} \mathrm{O}_{\text {atm }}\right)$ pouvaient être alignées directement sur les variations de $\delta^{18} \mathrm{O}_{\text {calcite }}$ des spéléothèmes des basses latitudes ce qui permet de réduire les barres d'erreur de datation (Extier et al., 2018). Cette possibilité d'aligner le $\delta^{18} \mathrm{O}_{\text {atm }}$ sur le $\delta^{18} \mathrm{O}_{\text {calcite }}$ des spéléothèmes fait du $\delta^{18} \mathrm{O}_{\text {atm }}$ un nouvel outil enrichissant la palette de la synchronisation aux spéléothèmes. 


\section{Les paramètres orbitaux de la Terre}

Variations de l'EXCENTRICIT É de l'orbite terrestre
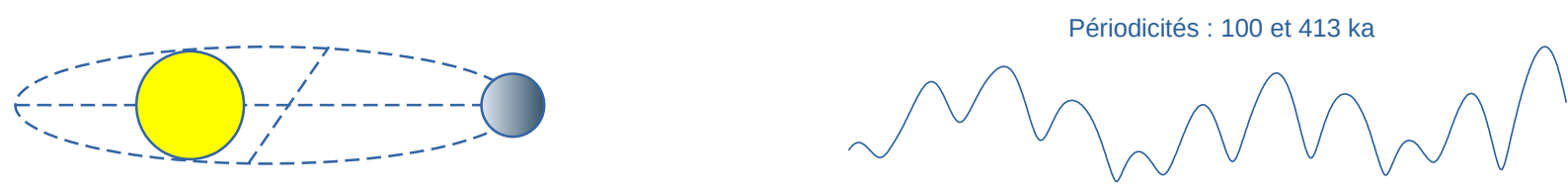

Variations de l'OBLIQUIT É de l'axe de rotation
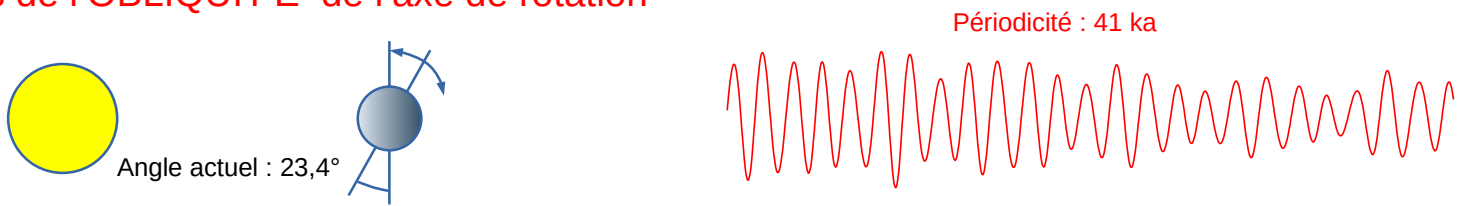

\section{PR É CESSION de l'axe de rotation et ROTATION de l'orbite}
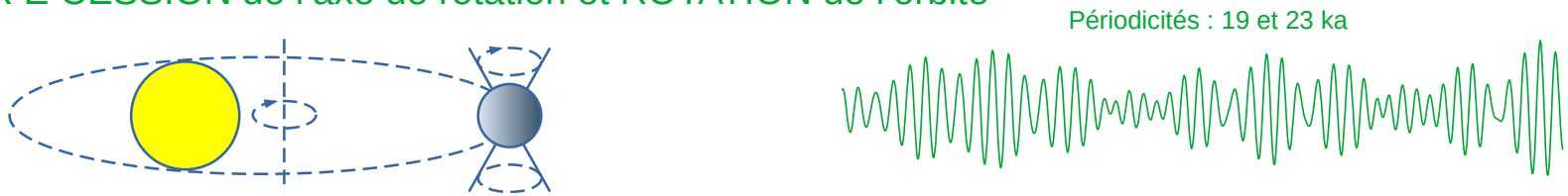

Il y a trois paramètres orbitaux qui influencent la quantité d'énergie solaire reçue par la Terre à une latitude et à une date donnée. L'orbite terrestre a une forme d'ellipse dont le soleil est un foyer. L'excentricité mesure l'aplatissement de cette ellipse, sachant que le grand axe de l'ellipse est quasi-constant et que c'est le petit axe qui varie. Cette excentricité a varié entre 0 et $6 \%$ environ lors du dernier million d'années. L'excentricité induit une forme de saisonnalité, en phase entre le nord et le sud : la Terre reçoit plus d'énergie quand elle est au plus proche du soleil (au périhélie) et moins lorsqu'elle est loin du soleil (à l'aphélie). L'obliquité est l'angle entre l'axe de rotation de la Terre et la perpendiculaire au plan orbital. Elle est actuellement de $23,4^{\circ}$ et a varié entre environ $22^{\circ}$ et $24,5^{\circ}$ au cours du dernier million d'années. L'obliquité induit les saisons que l'on connaît, avec un angle incident du soleil plus vertical et des jours plus longs en été. Les saisons sont en anti-phase entre le nord et le sud : c'est l'hiver dans le sud quand c'est l'été dans le nord, et réciproquement. L'angle de précession est l'angle entre le grand axe de l'ellipse et le projeté de l'axe de rotation de la Terre sur le plan orbital. Il conditionne la position de la Terre sur l'orbite au moment des saisons. Par exemple actuellement, la Terre se trouve au périhélie au début du mois de janvier, c'est-à-dire presque au solstice d'hiver de l'hémisphère nord et à l'aphélie au début du mois de juillet, c'est-à-dire presque au solstice d'été de l'hémisphère nord. Les saisons sont donc peu contrastées dans l'hémisphère nord, et très contrastées dans l'hémisphère sud. Dans les calculs d'insolation, c'est le paramètre de précession qui importe. Celui-ci est égal à l'excentricité multipliée par le sinus de l'angle de précession.

Les paramètres orbitaux varient au cours du temps, à cause de l'attraction des autres planètes du système solaire et à cause des couples mécaniques exercés par le soleil et la lune sur la Terre qui n'est pas parfaitement sphérique. L'excentricité varie avec des périodes principales de 100 et $413 \mathrm{ka}$ (milliers d'années). L'obliquité varie avec une période principale de $41 \mathrm{ka}$. La précession varie avec des périodes principales de 19 et $23 \mathrm{ka}$.

\section{Datation par calage sur les variations orbitales}

L'évolution des paramètres orbitaux de la Terre lors du dernier million d'années est connue avec une grande précision pour les derniers millions d'années (Laskar et al., 2004) et ces variations ont une signature dans la plupart des enregistrements climatiques. Il est donc naturel d'utiliser ces 
variations pour dater les carottes polaires. L'incertitude de cette datation tient d'une part à l'hypothèse d'un déphasage constant entre variations orbitales et variations climatiques et, d'autre part, à la détermination de ce déphasage. L'intérêt d'une telle datation est d'avoir une incertitude à peu près constante de quelques milliers d'années tout au long du forage, notamment pour les parties profondes, dès lors qu'on arrive encore à compter les cycles astronomiques.

Les enregistrements de plusieurs paramètres des carottes de glace présentent de fortes variations dans les fréquences astronomiques. Ils ont donc été utilisés pour le calage orbital des carottes de glace : ce sont le rapport $\mathrm{D} / \mathrm{H}$ de la glace, indicateur de la température locale et le rapport ${ }^{18} \mathrm{O} /{ }^{16} \mathrm{O}$ de l'air des bulles. Nous n'allons pas rentrer dans le détail des mécanismes complexes régissant les variations de ce dernier indicateur, mais nous dirons simplement que le $\delta^{18} \mathrm{O}_{\text {atm }}$ montre un lien très fort avec la précession sur les échelles des cycles astronomiques. Il est très facile de l'utiliser pour " compter » les cycles de précession, tout du moins pour les périodes pendant lesquelles ils sont bien marqués dans les variations astronomiques. Cependant, le déphasage entre le $\delta^{18} \mathrm{O}_{\text {atm }}$ et l'insolation est très probablement variable au cours du temps du fait de l'interaction entre la variabilité millénaire et la variabilité orbitale ce qui limite la précision de la contrainte chronologique obtenue avec cet outil.

Des indicateurs plus directs de l'insolation locale ont également été proposés pour compléter la palette d'outils de datation astronomique. En particulier, l'insolation locale en été modifie la structure de la neige en surface et cette signature reste présente jusqu'à la zone de fermeture. Cette modification de paramètres physiques dans le névé a un impact sur la teneur en air à la profondeur de piégeage et également sur des processus de fractionnement moléculaire entre $\mathrm{O}_{2}$ et $\mathrm{N}_{2}$ lorsque les pores se referment (la molécule d' $\mathrm{O}_{2}$ étant plus petite que celle de $\mathrm{N}_{2}$, elle est expulsée préférentiellement lors de la fermeture des pores).

C'est Bender (2002) qui a pour la première fois suggéré que le rapport $\mathrm{O}_{2} / \mathrm{N}_{2}$ des bulles d'air qu'il analysait dans la carotte Vostok dépendait de l'insolation locale au solstice d'été. Ce lien a été confirmé (Figure 7) dans la première carotte forée à Dôme Fuji (Kawamura et al., 2007). 


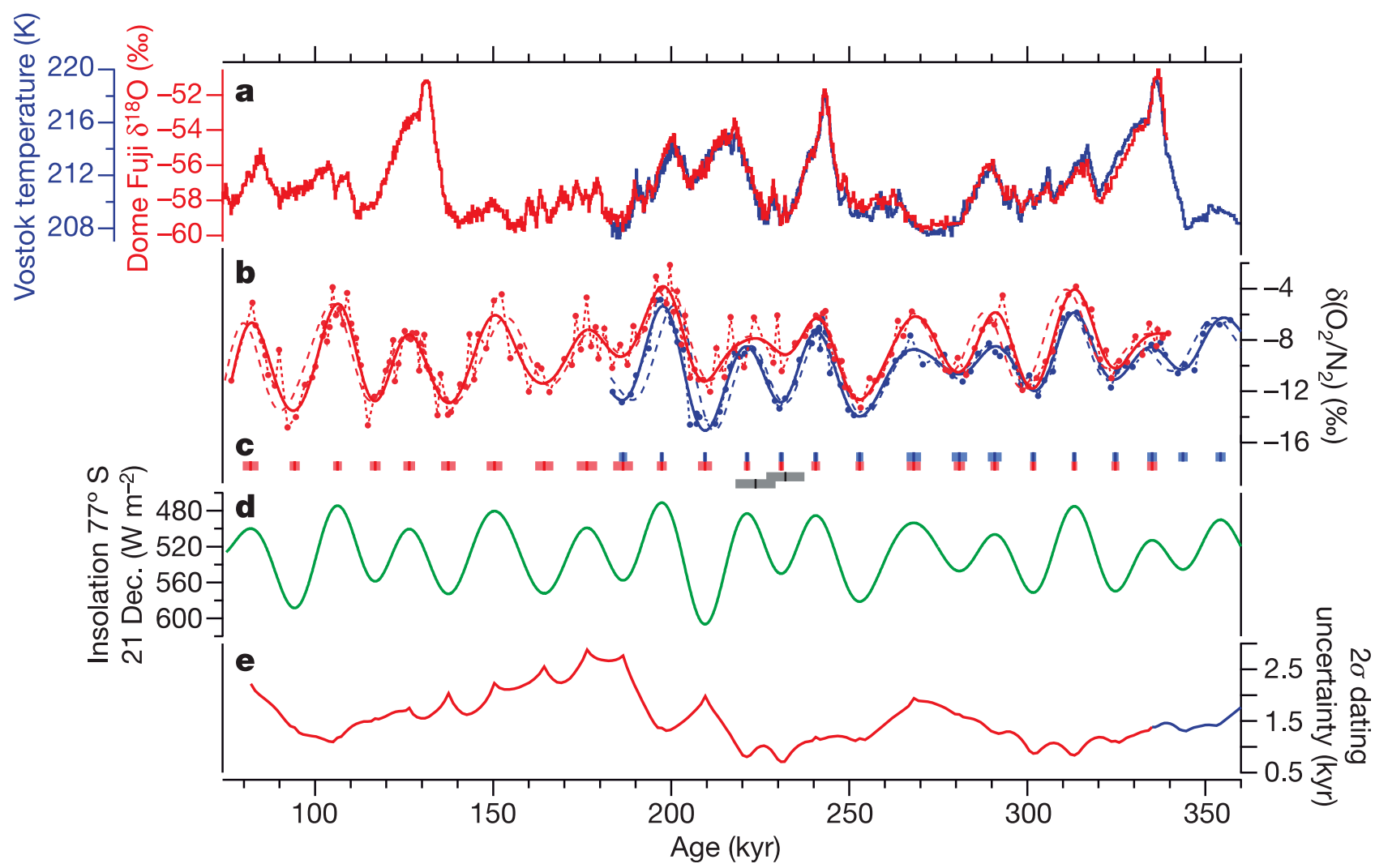

Figure 7 : Calage astronomique grâce à l'indicateur $\mathrm{O}_{2} / \mathrm{N}_{2}$. a) Composition isotopique de la glace et température à Vostok et Dôme Fuji. b) Rapport $\mathrm{O}_{2} / \mathrm{N}_{2}$ (indiqué en notation $\delta$ ) mesuré dans les bulles d'air à Dôme Fuji et Vostok. Les points sont les données brutes ; les lignes épaisses représentent les données filtrées. c) Marqueurs d'âge déduits du calage avec l'insolation locale, avec les barres d'erreur $2 \sigma$. d) Insolation du solstice d'été à $77^{\circ} \mathrm{S}$ utilisée comme cible de calage. e) Incertitude $2 \sigma$ de la datation $\mathrm{O}_{2} / \mathrm{N}_{2}$. Extrait de Kawamura et al. (2007).

En parallèle, Raynaud et al. (2007) ont montré que la teneur en air du forage EDC dépendait de l'insolation locale moyennée sur une période centrée sur le solstice d'été. Toutefois, pour ce deuxième indicateur, il faut également corriger des variations d'altitude à l'endroit de formation des bulles, car celles-ci ont un impact sur la pression atmosphérique et donc sur le contenu en air de ces bulles.

\section{La modélisation du processus de sédimentation}

La glace présente l'énorme avantage, par rapport à d'autres archives, de pouvoir être datée à l'aide de modèles physiques décrivant le processus de sédimentation glaciaire. L'âge de la glace et de l'air dans le forage à une profondeur peut ainsi être calculé à partir : 1) du taux d'accumulation initial de neige, 2) du rapport entre l'épaisseur d'une couche dans le forage et son épaisseur initiale (ce rapport étant appelé fonction d'amincissement) et 3) de la modélisation du processus de piégeage. Nous allons détailler plus bas le calcul de chacune de ces trois étapes.

\section{1. Évaluation de l'accumulation de surface}

Dans les premières centaines de mètres, l'amincissement des couches de neige et de glace est faible et bien évalué par la modélisation. On peut ainsi déterminer l'accumulation de surface à partir d'horizons bien datés comme les couches de cendres volcaniques (décrits dans la section 2.2). Audelà, on utilise en général la composition isotopique de la glace $\left(\mathrm{D} / \mathrm{H}\right.$ ou $\left.{ }^{18} \mathrm{O} /{ }^{16} \mathrm{O}\right)$. Toute comme 
pour la température de surface, les mesures de terrain en Antarctique et au Groenland montrent en effet une bonne corrélation de la composition isotopique avec l'accumulation de neige en surface. Cette corrélation provient de la dépendance de la pression de vapeur saturante à la température. Il faut noter toutefois que cette corrélation ne prend pas en compte les phénomènes de re-dépôt de la neige par le vent, qui modifient l'accumulation sans forcément altérer la composition isotopique de la neige. D'autre part, les phénomènes cycloniques locaux vont affecter cette relation. Enfin, lorsque l'on extrapole cette relation aux variations temporelles d'accumulation, on doit tenir compte des variations de température et de composition isotopique à la source des masses d'air qui modifient également la composition isotopique de la glace (Uemura et al., 2012).

\subsection{Modèles d'écoulement de la glace}

La glace est un solide au comportement viscoplastique dont on peut déterminer expérimentalement et théoriquement la relation entre contrainte et déformation. On peut ainsi simuler la trajectoire suivie au cours du temps par une particule de glace au sein du glacier pour aboutir à une chronologie. La modélisation du comportement de la glace au sein d'un inlandsis requiert non seulement une bonne connaissance des propriétés viscoplastiques du matériau, mais aussi des conditions aux limites de la calotte. Ces conditions aux limites sont 1) la température et l'accumulation de surface au cours du temps ; 2) les conditions basales, comme le flux géothermique ou le taux de frottement sur le lit rocheux ; 3) les conditions latérales du domaine considéré, puisqu'on utilise pour la datation des modèles locaux au voisinage des sites de forage. Ces conditions latérales sont généralement issues de simulations globales de la calotte polaire au cours du temps (Ritz et al., 2001). On obtient ainsi la fonction d'amincissement du forage.

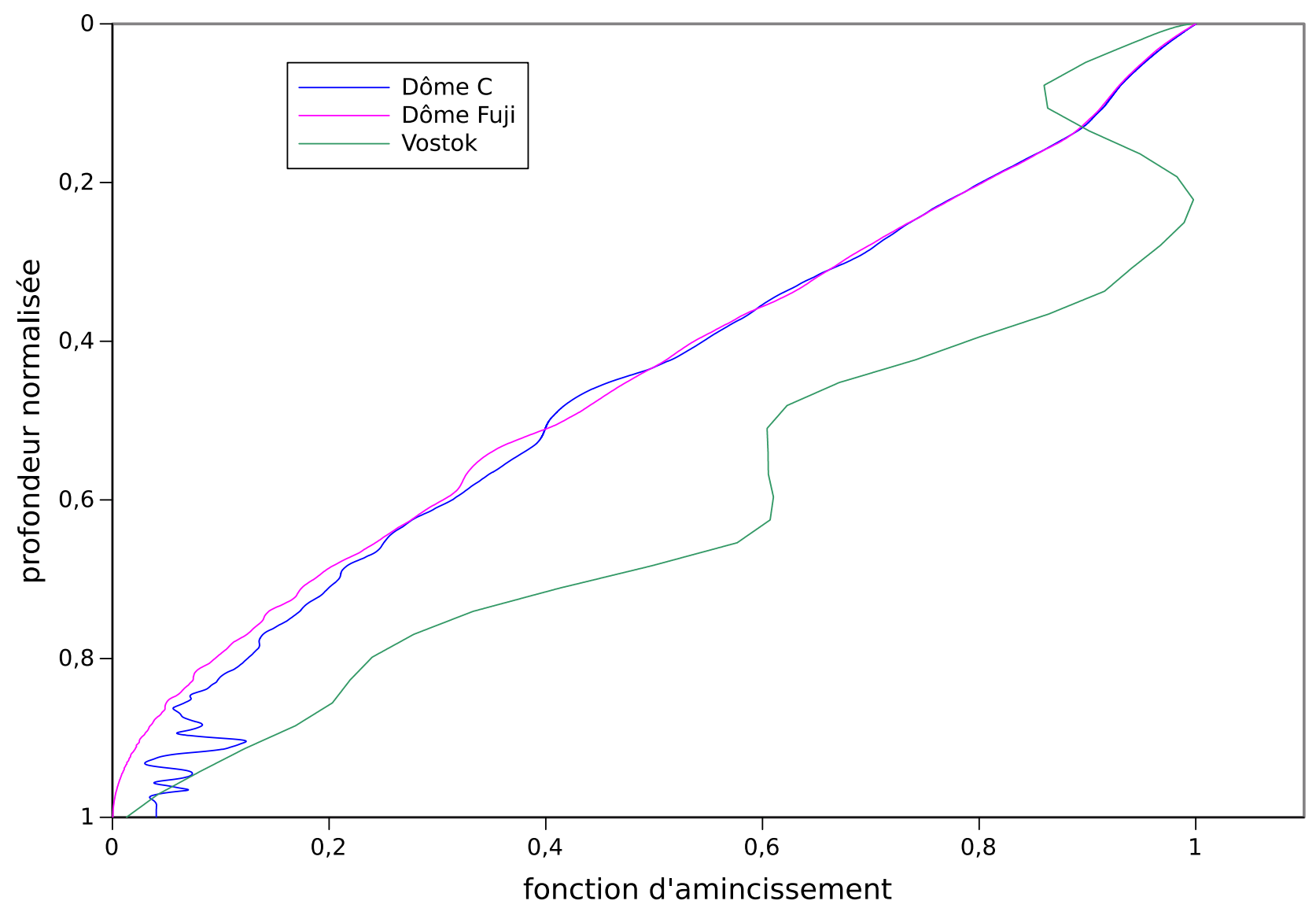

Figure 8 : Fonctions d'amincissement estimées pour les forages à Dôme C, Dôme Fuji et Vostok (Parrenin, 2013). 


\subsection{Modélisation du piégeage de l'air}

Pour évaluer le décalage glace/air ( $\Delta$ âge ou $\Delta$ profondeur), on doit évaluer :

- la densité du névé à la fermeture.

- l'âge de l'air à la fermeture, évalué grâce à un modèle de diffusion des gaz dans le névé (Buizert et al., 2012).

- le profil de densité dans le névé, qui est déduit d'un modèle mécanique. Différents modèles mécaniques ont été publiés (voir par exemple Bréant et al., 2017). Ces modèles prennent généralement en compte a) le glissement des grains de neige les uns par rapport aux autres, processus qui est dominant en surface, $b$ ) la déformation des grains qui devient dominante en profondeur.

Comme le montre la Figure 9, la profondeur de fermeture calculée augmente lorsque l'accumulation augmente (l'advection verticale augmente) ou lorsque la température diminue (la densification est alors moins rapide).

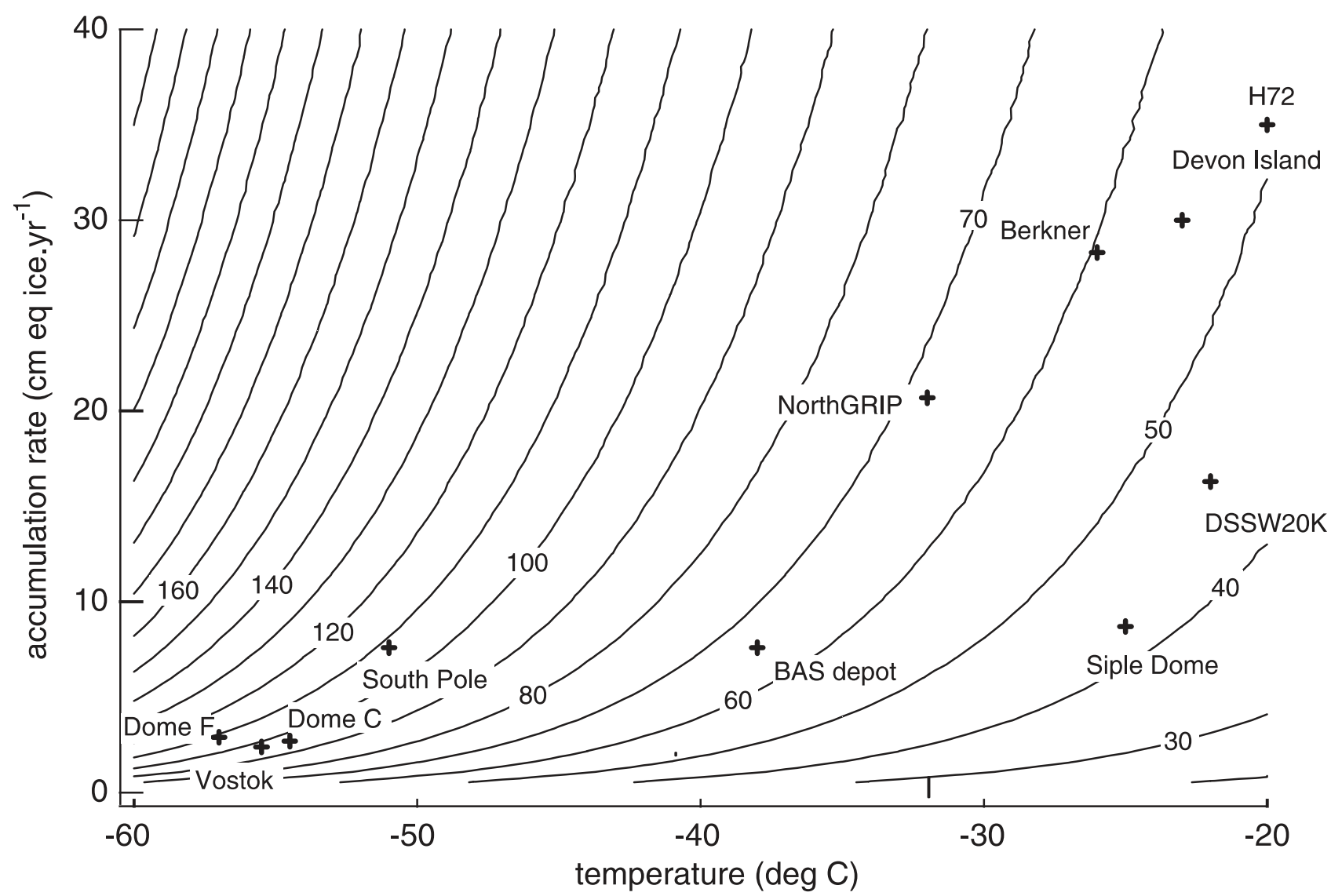

Figure 9 : Profondeur (en mètres) à la fermeture des bulles (courbes pleines) en fonction du taux d'accumulation et de la température de surface, comme calculée par le modèle d'Arnaud et al. (2000) supposé en état stationnaire. Les conditions en différents sites polaires sont indiquées par les croix. Extrait de Landais et al. (2006).

Alternativement au modèle de névé, on peut utiliser les isotopes de l'azote pour évaluer la hauteur du névé. L'azote a une composition isotopique (exprimée comme $\delta^{15} \mathrm{~N}$ ) à peu près constante dans l'atmosphère aux échelles de temps étudiées dans les carottes de glace. Cependant, la composition isotopique de l'azote dans les bulles d'air se modifie en raison de processus se déroulant dans le névé.

Aucun fractionnement ne se produit dans la zone convective en raison du brassage. Dans la zone 
diffusive, deux types de fractionnement ont lieu :

- le fractionnement gravitationnel entraîne, sous l'effet du champ de pesanteur, les isotopes lourds vers le fond du névé. Ce fractionnement dépendra donc principalement de la hauteur de la colonne diffusive, et dans un moindre mesure de la température du névé.

- le fractionnement thermique entraîne les espèces les plus lourdes vers l'extrémité froide. Ce fractionnement a lieu uniquement quand la température est différente aux deux extrémités de la colonne diffusive, c'est-à-dire principalement pendant les changements de température abrupts (plusieurs degrés en une centaine d'années).

Le $\delta^{15} \mathrm{~N}$ de l'air piégé dans les bulles d'air peut ainsi être utilisé de deux manières différentes pour contraindre le $\Delta$ profondeur.

Premièrement, les variations abruptes de température peuvent être repérées à la fois dans la phase solide (où elles sont enregistrées dans les variations de la composition isotopique de la molécule $\mathrm{H}_{2} \mathrm{O}$ ) et dans la phase gaz (grâce à l'anomalie de composition isotopique due au fractionnement thermique) et ce à des profondeurs différentes, donnant ainsi une mesure du $\Delta$ profondeur.

Deuxièmement, en l'absence de changement abrupt de température on peut déduire l'épaisseur de la colonne diffusive des valeurs de $\delta^{15} \mathrm{~N}$ à partir de la formule du fractionnement gravitationnel.

\subsection{Les limites de la modélisation}

Malheureusement, l'exercice de datation par modélisation devient de plus en plus imprécis à mesure que l'on s'approche de la base de la calotte, pour diverses raisons. Premièrement, les propriétés mécaniques de la glace ne sont pas parfaitement connues. Elles dépendent non seulement des conditions de pression et de température, mais aussi de la taille et de l'orientation des cristaux qui forment cette glace. Deuxièmement, les conditions à la base du socle rocheux ne peuvent pas être mesurées directement in situ. Enfin, les conditions latérales au cours du passé, issues du modèle à grande échelle, peuvent également être entachées d'une erreur importante. Ces conditions latérales conditionnent la position des dômes et lignes de partage sur le domaine, et donc le trajet des particules de glace.

\section{La méthode inverse : une approche fédérative}

Toutes les méthodes de datation décrites dans les sections précédentes ont des avantages et des inconvénients. Ces différentes sources d'information chronologique étant complémentaires, il est donc clair que l'obtention d'une datation optimale des archives glaciaires nécessite de les combiner. C'est pourquoi une méthode d'optimisation a été développée (IceChrono, Parrenin et al., 2015). Celle-ci inverse les trois variables du modèle de sédimentation (taux d'accumulation, profondeur de piégeage et fonction d'amincissement) et s'applique à plusieurs forages simultanément, en prenant en compte les liens stratigraphiques entre ces forages, les horizons datés, les intervalles datés, ainsi que les observations du $\Delta$ profondeur (Figure 10). Cette méthode a été ensuite appliquée pour obtenir une chronologie de référence, nommée AICC2012, pour 4 carottes d'Antarctique (EDC, EDML, Vostok, TALDICE) en accord avec la chronologie GICC05 de NGRIP au Groenland (Bazin et al., 2013; Veres et al., 2013). 


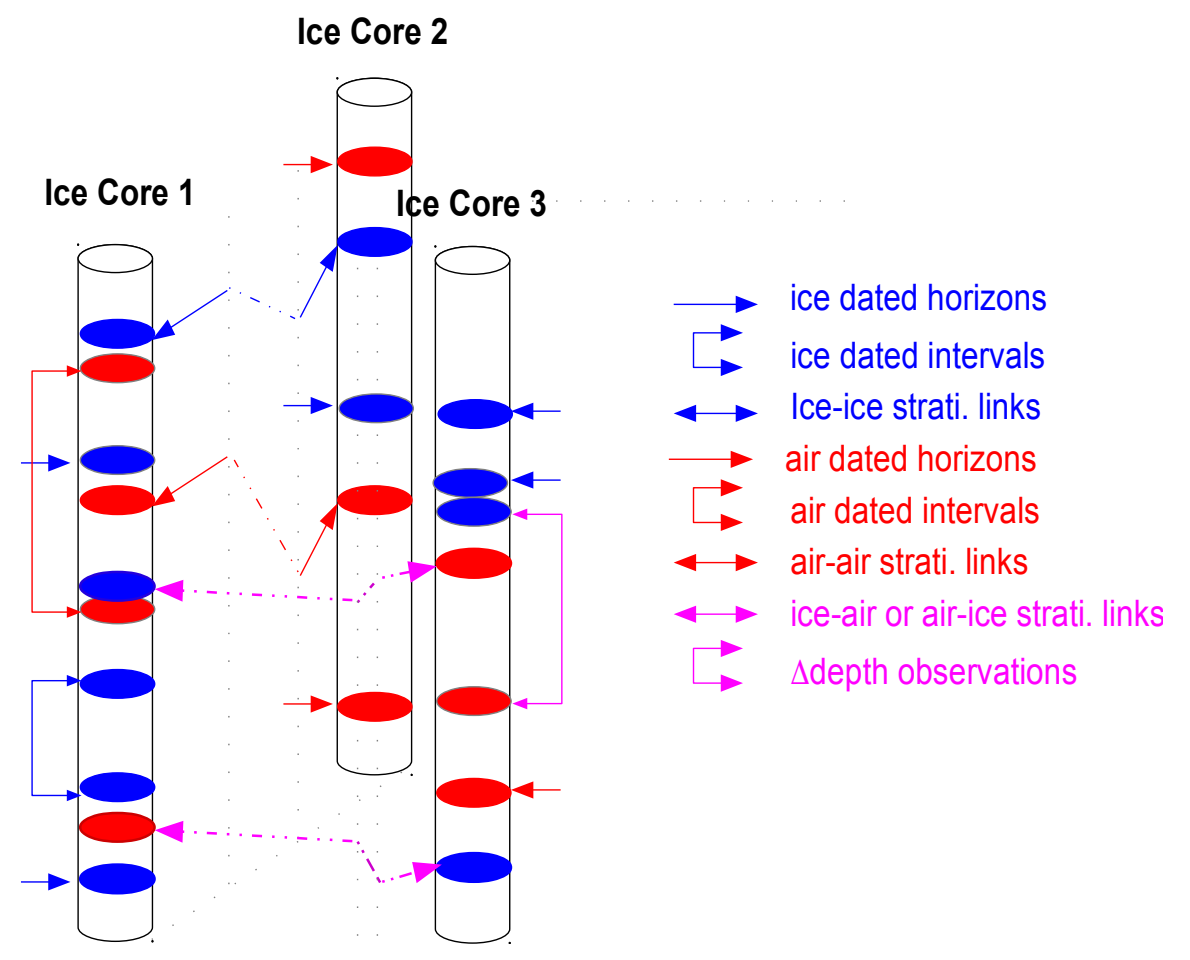

Figure 10: Les différentes types d'information chronologique utilisés par le modèle probabiliste IceChrono pour optimiser conjointement la datation de plusieurs forages glaciaires. Extrait de Parrenin et al. (2015).

\section{Conclusion}

La datation des archives glaciaires est donc un problème complexe qui, en l'absence de méthodes radioactives précises, est basé sur plusieurs techniques complémentaires. Pour l'Holocène et au Groenland, la datation par comptage des couches GICC05 est précise à environ 1\% près. Cette datation a été confirmée par comparaison à la dendrochronologie grâce au ${ }^{10} \mathrm{Be}$ ou à des horizons volcaniques datés par ${ }^{14} \mathrm{C}$. En Antarctique, le dernier millénaire peut être daté à quelques années près par reconnaissance d'éruptions volcaniques. Pour le reste de l'Holocène, la synchronisation à la datation du Groenland ou à l'échelle dendrochronologique grâce au ${ }^{10} \mathrm{Be}$ nous fournit une datation à quelques dizaines d'années près.

Pour la dernière période glaciaire, la précision du comptage des couches annuelles décroît à environ $5 \%$ jusqu'à $60 \mathrm{ka}$. Cette datation fournit des âges pour les événements de DansgaardOeschger qui sont confirmés par les datations uranium/thorium de spéléothèmes d'Europe et d'Asie. Elle est également compatible avec quelques horizons volcaniques datés par ${ }^{14} \mathrm{C}$ ou argon/argon. Les principales méthodes de datation pour les derniers $60 \mathrm{ka}$ semblent donc maintenant être en accord à quelques centaines d'années, plutôt que quelques milliers d'années comme c'était le cas il y a 20 ans. Cette datation est transposée sur les carottes Antarctique grâce à la synchronisation méthane. Cependant, cette démarche nécessite l'évaluation du $\Delta$ âge et celui-ci est incertain pour les périodes glaciaires en Antarctique central.

Au-delà de la dernière période glaciaire, le comptage des couches n'est plus possible, les spéléothèmes ou les horizons bien datés deviennent très rares et les datations sont principalement basées sur le calage astronomique. Ce dernier n'est précis qu'à environ 5000 ans près avec les méthodes classiques basées sur des enregistrements climatiques. Les indicateurs d'insolation locale 
(rapport $\mathrm{O}_{2} / \mathrm{N}_{2}$, teneur en air) pourrait permettre de descendre à une précision de 1 à 2 ka mais ceci doit être confirmé par des méthodes indépendantes. De nouvelles spéléothèmes couvrant ces périodes plus anciennes ont également vu le jour et apportent de nouvelles contraintes via l'enregistrement en ${ }^{18} \mathrm{O}$ des bulles d'air. Dans ce cas-là il faudra utiliser la datation Uranium/Plomb pour les spéléothèmes (Woodhead and Petrus, 2019).

La construction d'une datation optimale sera un des grands chantiers du grand projet européen de forage Beyond EPICA qui vise à obtenir une carotte de glace couvrant les derniers 1.5 million d'années en Antarctique. Ce projet permettra ainsi de mieux comprendre la transition du miPléistocène survenue il y a environ 1 million d'années et durant laquelle les cycles glaciairesinterglaciaires sont devenues plus amples et avec une période d'environ 100000 ans, contre environ 40000 ans auparavant.

\section{Références :}

Arnaud, L., Barnola, J.-M. and Duval, P.: Physical modeling of the densification of snow/firn and ice in the upper part of polar ice sheets, in Physics of Ice Core Records, edited by T. Hondoh, pp. 285-305, Hokkaido University Press, Sapporo, Japan., 2000.

Bazin, L., Landais, A., Lemieux-Dudon, B., Toyé Mahamadou Kele, H., Veres, D., Parrenin, F., Martinerie, P., Ritz, C., Capron, E., Lipenkov, V., Loutre, M.-F., Raynaud, D., Vinther, B., Svensson, A., Rasmussen, S. O., Severi, M., Blunier, T., Leuenberger, M., Fischer, H., MassonDelmotte, V., Chappellaz, J. and Wolff, E.: An optimized multi-proxy, multi-site Antarctic ice and gas orbital chronology (AICC2012): 120-800 ka, Clim. Past, 9(4), 1715-1731, doi:10.5194/cp-91715-2013, 2013.

Beer, J., Vonmoos, M. and Muscheler, R.: Solar Variability Over the Past Several Millennia, Space Science Reviews, 125(1), 67-79, doi:10.1007/s11214-006-9047-4, 2006.

Bender, M. L.: Orbital tuning chronology for the Vostok climate record supported by trapped gas composition, Earth Planet. Sci. Lett., 204, 275-289, 2002.

Bender, M. L., Barnett, B., Dreyfus, G., Jouzel, J. and Porcelli, D.: The contemporary degassing rate of ${ }^{40} \mathrm{Ar}$ from the solid Earth, PNAS, 105(24), 8232-8237, doi:10.1073/pnas.0711679105, 2008.

Bréant, C., Martinerie, P., Orsi, A., Arnaud, L. and Landais, A.: Modelling firn thickness evolution during the last deglaciation: constraints on sensitivity to temperature and impurities, Climate of the Past, 13(7), 833-853, doi:https://doi.org/10.5194/cp-13-833-2017, 2017.

Buizert, C., Martinerie, P., Petrenko, V. V., Severinghaus, J. P., Trudinger, C. M., Witrant, E., Rosen, J. L., Orsi, A. J., Rubino, M., Etheridge, D. M. and Others: Gas transport in firn: multipletracer characterisation and model intercomparison for NEEM, Northern Greenland, Atmos. Chem. Phys, 12, 4259-4277, 2012.

Buizert, C., Baggenstos, D., Jiang, W., Purtschert, R., Petrenko, V. V., Lu, Z.-T., Müller, P., Kuhl, T., Lee, J., Severinghaus, J. P. and Brook, E. J.: Radiometric ${ }^{81} \mathrm{Kr}$ dating identifies 120,000-year-old ice at Taylor Glacier, Antarctica, Proceedings of the National Academy of Sciences, 111(19), 68766881, 2014.

EPICA community members: One-to-one coupling of glacial climate variability in Greenland and Antarctica, Nature, 444, 195-198, 2006. 
Extier, T., Landais, A., Bréant, C., Prié, F., Bazin, L., Dreyfus, G., Roche, D. M. and Leuenberger, M.: On the use of $\delta^{18} \mathrm{O}_{\text {atm }}$ for ice core dating, Quaternary Science Reviews, 185, 244-257, doi:10.1016/j.quascirev.2018.02.008, 2018.

Guillou, H., Singer, B. S., Laj, C., Kissel, C., Scaillet, S. and Jicha, B. R.: On the age of the Laschamp geomagnetic excursion, Earth Planet. Sci. Lett., 227, 331-343, 2004.

Johnsen, S. J., Clausen, H. B., Dansgaard, W., Fuhrer, K., Gundestrup, N., Hammer, C. U., Iversen, P., Jouzel, J., Stauffer, B. and Steffensen, J. P.: Irregular glacial interstadials recorded in a new Greenland ice core, Nature, 359, 311-313, 1992.

Kawamura, K., Parrenin, F., Uemura, R., Vimeux, F., Severinghaus, J. P., Matsumoto, K., Nakata, H., Nakazawa, T., Aoki, S., Jouzel, J., Fujii, Y. and Watanabe, O.: Northern Hemisphere forcing of climatic cycles over the past 360,000 years implied by absolute dating of Antarctic ice cores, Nature, 448, 912-917, doi:10.1038/nature06015, 2007.

Landais, A., Barnola, J. M., Kawamura, K., Caillon, N., Delmotte, M., Ommen, T. V., Dreyfus, G., Jouzel, J., Masson-Delmotte, V., Minster, B., Freitag, J., Leuenberger, M., Schwander, J., Huber, C., Etheridge, D. and Morgan, V.: Firn-air $\delta^{15} \mathrm{~N}$ in modern polar sites and glacial-interglacial ice: a model-data mismatch during glacial periods in Antarctica?, Quat. Sci. Rev., 25(1-2), 49-62, doi:DOI: 10.1016/j.quascirev.2005.06.007, 2006.

Laskar, J., Robutel, P., Joutel, F., Gastineau, M., Correia, A. C. M. and Levrard, B.: A long-term numerical solution for the insolation quantities of the Earth, Astronomy and Astrophysics, 428, 261-285, doi:10.1051/0004-6361:20041335, 2004.

Parrenin, F.: Sur l'âge de la glace et des bulles d'air dans les calottes polaires, Université Joseph Fourier, Grenoble, France., 2013.

Parrenin, F., Bazin, L., Capron, E., Landais, A., Lemieux-Dudon, B. and Masson-Delmotte, V.: IceChrono1: a probabilistic model to compute a common and optimal chronology for several ice cores, Geosci. Model Dev., 8(5), 1473-1492, doi:10.5194/gmd-8-1473-2015, 2015.

Raisbeck, G. M., Yiou, F., Jouzel, J. and Stocker, T. F.: Direct north-south synchronization of abrupt climate change record in ice cores using Beryllium 10, Clim. Past, 3(3), 541-547, 2007.

Rasmussen, S. O., Andersen, K. K., Svensson, A. M., Steffensen, J. P., Vinther, B. M., Clausen, H. B., Siggaard-Andersen, M.-L., Johnsen, S. J., Larsen, L. B., Dahl-Jensen, D., Bigler, M.,

Rothlisberger, R., Fischer, H., Goto-Azuma, K., Hansson, M. E. and Ruth, U.: A new Greenland ice core chronology for the last glacial termination, J. Geophys. Res., 111, D06102, doi:10.1029/2005JD006079, 2006.

Raynaud, D., Lipenkov, V., Lemieux-Dudon, B., Duval, P., Loutre, M.-F. and Lhomme, N.: The local insolation signature of air content in Antarctic ice. A new step toward an absolute dating of ice records, Earth Planet. Sci. Lett., 261(3-4), 337-349, 2007.

Reimer, P. J., Bard, E., Bayliss, A., Beck, J. W., Blackwell, P. G., Ramsey, C. B., Buck, C. E., Cheng, H., Edwards, R. L., Friedrich, M. and others: IntCal13 and Marine13 radiocarbon age calibration curves 0-50,000 years cal BP, Radiocarbon, 55(4), 1869-1887, 2013.

Ritz, C., Rommelaere, V. and Dumas, C.: Modeling the evolution of Antarctic ice sheet over the last 420,000 years: Implications for altitude changes in the Vostok region, J. Geophys. Res., 
Sagnotti, L., Giaccio, B., Liddicoat, J. C., Nomade, S., Renne, P. R., Scardia, G. and Sprain, C. J.: How fast was the Matuyama-Brunhes geomagnetic reversal? A new subcentennial record from the Sulmona Basin, central Italy, Geophys J Int, 204(2), 798-812, doi:10.1093/gji/ggv486, 2016.

Sigl, M., McConnell, J. R., Toohey, M., Curran, M., Das, S. B., Edwards, R., Isaksson, E., Kawamura, K., Kipfstuhl, S., Krüger, K., Layman, L., Maselli, O. J., Motizuki, Y., Motoyama, H., Pasteris, D. R. and Severi, M.: Insights from Antarctica on volcanic forcing during the Common Era, Nature Climate Change, 4(8), 693-697, 2014.

Sigl, M., Fudge, T. J., Winstrup, M., Cole-Dai, J., Ferris, D., McConnell, J. R., Taylor, K. C., Welten, K. C., Woodruff, T. E., Adolphi, F., Bisiaux, M., Brook, E. J., Buizert, C., Caffee, M. W., Dunbar, N. W., Edwards, R., Geng, L., Iverson, N., Koffman, B., Layman, L., Maselli, O. J., McGwire, K., Muscheler, R., Nishiizumi, K., Pasteris, D. R., Rhodes, R. H. and Sowers, T. A.: The WAIS Divide deep ice core WD2014 chronology - Part 2: Annual-layer counting (0-31 ka BP), Clim. Past, 12(3), 769-786, doi:10.5194/cp-12-769-2016, 2016.

Sowers, T. A., Bender, M., Raynaud, D. and Korotkevich, Y. L.: The $\delta^{15} \mathrm{~N}$ of $\mathrm{N}_{2}$ in air trapped in polar ice : a tracer of gas transport in the firn and a possible constraint on ice age-gas age differences, J. Geophys. Res., 97(15), 15683-15697, 1992.

Svensson, A., Andersen, K. K., Bigler, M., Clausen, H. B., Dahl-Jensen, D., Davies, S. M., Johnsen, S. J., Muscheler, R., Parrenin, F., Rasmussen, S. O., Röthlisberger, R., Seierstad, I., Steffensen, J. P. and Vinther, B. M.: A 60000 year Greenland stratigraphic ice core chronology, Clim. Past, 4(1), 47-57, 2008.

Uemura, R., Masson-Delmotte, V., Jouzel, J., Landais, A., Motoyama, H. and Stenni, B.: Ranges of moisture-source temperature estimated from Antarctic ice cores stable isotope records over glacialinterglacial cycles, Clim. Past, 8(3), 1109-1125, doi:10.5194/cp-8-1109-2012, 2012.

Veres, D., Bazin, L., Landais, A., Toyé Mahamadou Kele, H., Lemieux-Dudon, B., Parrenin, F., Martinerie, P., Blayo, E., Blunier, T., Capron, E., Chappellaz, J., Rasmussen, S. O., Severi, M., Svensson, A., Vinther, B. and Wolff, E. W.: The Antarctic ice core chronology (AICC2012): an optimized multi-parameter and multi-site dating approach for the last 120 thousand years, Clim. Past, 9(4), 1733-1748, doi:10.5194/cp-9-1733-2013, 2013.

Wang, X., Auler, A. S., Edwards, L., Cheng, H., Cristalli, P. S., Smart, P. L., Richards, D. A. and Shen, C.-C.: Wet periods in northeastern Brazil over the past $210 \mathrm{kyr}$ linked to distant climate anomalies, Nature, 432, 740-743, 2004.

Wang, Y. J., Cheng, H., Edwards, R. L., An, Z. S., Wu, J. Y., Shen, C. C. and Dorale, J. A.: A highresolution absolute-dated late Pleistocene Monsoon record from Hulu Cave, China., Science, 294(5550), 2345-2348, 2001.

Woodhead, J. and Petrus, J.: Exploring the advantages and limitations of in situ U-Pb carbonate geochronology using speleothems, Geochronology, 1(1), 69-84, doi:10.5194/gchron-1-69-2019, 2019. 